\title{
Doubling of optical signals using triangular pulses
}

\author{
Anton I. Latkin,, ${ }^{1,2}$ Sonia Boscolo, ${ }^{2, *}$ Ranjeet S. Bhamber, ${ }^{2}$ and Sergei K. Turitsyn ${ }^{2}$ \\ ${ }^{1}$ Novosibirsk State University, Pirogova Street 2, Novosibirsk 630090, Russia \\ ${ }^{2}$ Photonics Research Group, School of Engineering and Applied Science, Aston University, Birmingham B4 7ET, \\ United Kingdom \\ *Corresponding author: s.a.boscolo@aston.ac.uk
}

Received February 10, 2009; revised June 4, 2009; accepted June 5, 2009; posted June 9, 2009 (Doc. ID 107328); published July 7, 2009

\begin{abstract}
We propose a novel technique of doubling optical pulses in both frequency and time domains based on a combination of cross-phase modulation induced by a triangular pump pulse in a nonlinear Kerr medium and subsequent propagation in a dispersive medium. (C) 2009 Optical Society of America

OCIS codes: $190.0190,070.4340,060.2310$.
\end{abstract}

\section{INTRODUCTION}

All-optical signal processing holds the key to unlocking the major advantages of photonics over electronics in the field of telecommunications such as large bandwidth and high-speed communication. However, despite recent efforts, electronic switches and routers still limit the information processing speed of current fiber communication systems. Electronic techniques of signal manipulation are still advanced compared to the first pioneering all-optical processing devices. Fundamentally, optical signal processing is still very much a research area, and considerable knowledge has still to be accumulated before a true breakthrough can be achieved in this field. An important advantage of electronics is the availability of a variety of simple, elementary pulse shapes that provide the foundations for a range of basic logical and analog operations. Matching a range of electronic functions in the optical domain is one of the most important research challenges. Nonlinear photonics is widely considered to be the key to the implementation of all-optical devices capable of performing analog signal processing and a variety of advanced digital operations. In this paper, we propose the use of recently introduced triangular optical pulses [1] to realize the function of all-optical pulse doubling in both the frequency and time domains. Beyond optical signal processing, the proposed technique can be used effectively in all-optical signal regeneration and can be of interest in laser physics, in addition to other applications that require controllable frequency conversion and pulse doubling.

Manipulation of optical pulses using cross-phase modulation (XPM) by a control pulse in a nonlinear Kerr medium is a well-established technique for compression and switching of optical pulses in the time and/or frequency domain [2]. This technique is rather generic and can be used in many physical problems. Within the pump-probe configuration, which is often used in the case of optical fibers [3], a nonlinear phase shift is induced on the weak signal wave pulse by a copropagating strong pump wave pulse through XPM, which is proportional to the temporal intensity of the pump pulse. The temporal and spectral ef- fects of XPM on the evolution of copropagating pulses in an optical fiber has been extensively studied over the past two decades (see, for example, [4-7]). In [6] the emphasis was given to the defocusing situation where the groupvelocity dispersion (GVD) and nonlinearity of the signal pulse have opposite signs. It was demonstrated that in such a situation the pump splits the signal pulse into two smaller (frequency-shifted) pulse fragments that separate in time. The key idea of our approach is to take advantage of the simple form of a triangular pulse (used as the control pump) to achieve controllable and analytically described doubling of a signal pulse.

Controlling the shape of optical pulses has become increasingly important for many scientific applications, including ultra-high-speed optical telecommunications and computing systems, quantum optics, and nonlinear optics. The possibility of changing the pulse waveform from the well-known Gaussian and hyperbolic secant shapes to more exotic parabolic or triangular pulses can be used for various applications in optical signal processing and manipulation. In [8], various transform-limited pulse shapes were generated by temporal coherent synthesization using a multi-arm interferometer. Generation of flat-top-[9], parabolic-[10,11], and sawtooth-(asymmetric triangular) [12] shaped pulses has been demonstrated using superstructured fiber Bragg grating technology. Flat-top, parabolic, and triangular pulses can also be produced through the use of nonlinear effects in optical fibers. We have recently introduced a method for passive nonlinear pulse shaping in the time domain, which relies on a combination of pulse prechirping and nonlinear propagation in a fiber with normal GVD [1]. Such recent advances in pulse shaping have paved the way for tailoring the phase profile imposed on a pulse using XPM or self-phase modulation (SPM). For instance, parabolic pulses yielding a linear frequency chirp (first time derivative of the phase) have been used for pulse retiming [10] as well as in the time domain optical Fourier transform technique [13] to mitigate signal waveform distortions caused by linear perturbations in optical fibers [14]. Sawtooth pulses inducing a constant chirp have been shown to enhance the perfor- 
mance of wavelength converters based on SPM in fiber and offset filtering [12]. In [15], XPM with triangular pump pulses was used to realize time-to-frequency mapping of multiplexed signals in high-speed fiber communication systems.

In this paper, we propose an all-optical technique of doubling optical pulses in both the frequency and time domains, that relies on a combination of XPM induced by a triangular pump pulse in a nonlinear Kerr medium and subsequent propagation in a linear dispersive medium. The constant, distinct (opposite sign) frequency shift (chirp) induced onto the leading (down-shifted) and trailing (up-shifted) edges of the signal pulse by the triangular pump wave allows, under certain conditions, an approximate replication of the signal pulse shape in the spectral domain, which is subject to a scaling of the peak intensity and width. Under subsequent propagation of the modulated signal pulse in a dispersive medium, the produced spectral separation of the pulse components leads to their subsequent separation in time, thus allowing temporal replication of the pulse regardless of the sign of the dispersion.

\section{SPECTRAL DOUBLING}

First, we focus on the spectral doubling of optical pulses. We consider the copropagation of a Gaussian signal pulse $\psi(0, t)=u_{0} \exp \left(-t^{2} /\left(2 T^{2}\right)\right)$ and a triangular pump pulse $\chi(0, t)=v_{0}\left(1-\left|t / T_{T}\right|\right)^{1 / 2} \theta\left(T_{T^{-}}|t|\right)$ in a nonlinear fiber with the nonlinear Kerr coefficient $\sigma$ and the length $L$. Here, $\theta(x)$ is the Heaviside function, and the case of temporally cocentered pulses (under the condition that their spectra do not overlap) is considered. In many experimental situations the fiber length is small compared with both the length scales associated with the two pulses where the GVD and dispersive walk-off effects take place. The dispersive terms in the corresponding coupled-amplitude nonlinear Schrödinger (NLS) equations inside the fiber [2] can then be neglected. Furthermore, in the situation of a strong pump pulse interacting with a weak signal pulse $\left(\left|v_{0}\right|^{2} \gg\left|u_{0}\right|^{2}\right)$, the SPM of the signal pulse is negligible. Accordingly, the NLS equation of the signal pulse can be simplified as $\partial \psi / \partial z \approx i \sigma\left(2|\chi|^{2}\right) \psi$. Consequently, at the output of the fiber, the signal pulse will acquire a nonlinear phase shift dominated by the control pulse as $\phi(t)$ $=2 \sigma|\chi(0, t)|^{2} L=\phi_{0}\left(1-\left|t / T_{T}\right|\right) \theta\left(T_{T}-|t|\right)$, namely, the frequency shift $\partial \phi / \partial t$. The spectral amplitude of the resulting chirped pulse can be calculated analytically as

$$
\begin{aligned}
\tilde{\psi}(\Omega)= & \tilde{\psi}_{0}(\Omega) \exp \left(-\Omega^{2} / 2\right)+\tilde{\psi}_{-}(\Omega) \exp \left(-(\Omega-\Delta \Omega)^{2} / 2\right) \\
& +\tilde{\psi}_{+}(\Omega) \exp \left(-(\Omega+\Delta \Omega)^{2} / 2\right), \\
\tilde{\psi}_{0}(\Omega)= & \sqrt{2 \pi} u_{0} T\left[1+\frac{1}{2} \operatorname{erf}\left(\left(i \Omega-\eta^{-1}\right) / \sqrt{2}\right)-\frac{1}{2} \operatorname{erf}((i \Omega\right. \\
& \left.\left.\left.+\eta^{-1}\right) / \sqrt{2}\right)\right], \\
& \frac{1}{\tilde{\psi}_{-}(\Omega)=} \sqrt{2 \pi} u_{0} T \exp \left(i \phi_{0}\right)[\operatorname{erf}(i(\Omega-\Delta \Omega) / \sqrt{2})-\operatorname{erf}((i(\Omega \\
& \left.\left.\left.-\Delta \Omega)-\eta^{-1}\right) / \sqrt{2}\right)\right],
\end{aligned}
$$

$$
\tilde{\psi}_{+}(\Omega)=\tilde{\psi}_{-}(-\Omega), \eta=T / T_{T}, \Omega=\omega T, \Delta \Omega=\phi_{0} \eta .
$$

In Eq. (1), $\Omega$ is a normalized frequency, and $\operatorname{erf}(x)$ is the error function. The notable feature of Eq. (1) is that the central parts of the spectrum (described by the term proportional to $\tilde{\psi}_{0}$ ) are suppressed, whereas the outer parts (described by the terms proportional to $\tilde{\psi}_{\overline{+}}$ ) are enhanced and, as a consequence, the spectrum develops a structure consisting of two equal peaks. The separation $\Delta \Omega$ between the two spectral peaks increases as the product of the maximum phase shift $\phi_{0}$ and the "duty ratio" parameter $\eta$ (width of the signal pulse to the width of the pump pulse), while their form is almost preserved for $\eta$ sufficiently smaller than 1 . We note that for relatively large $\eta(\eta$ $\geqslant 0.5$ ) a low-intensity multipeak structure forms between the two main peaks. Examples of spectral intensity profiles for different values of $\phi_{0}$ and $\eta=0.35$ are shown in Fig. 1 (top). The initial pulse spectrum is also plotted for comparison. It can be seen in Fig. 1 (top) that the maximum point of the spectral power of the two formed peaks is approximately four times as small as the maximum point of the initial spectrum, and the width of the peaks is approximately two times larger than that of the initial spectrum. This is consistent with conservation of the total energy. A closer inspection of function (1) under the assumption of small duty ratio parameter $(\eta \ll 1)$ reveals that in the proximity of the center of the pulse spectrum, i.e., when $\Omega \leqslant 1$,

$$
\widetilde{\psi}_{0}(\Omega) \simeq u_{0} T \eta \exp \left(-1 /\left(2 \eta^{2}\right)\right) \cos (\Omega / \eta),
$$

i.e., the central parts of the spectrum completely vanish. On the other hand, in the proximity of the center of either side peak, i.e., when $\epsilon=\Omega \mp \Delta \Omega \leqslant 1$, the form of the peak can be described by the following series expansion:

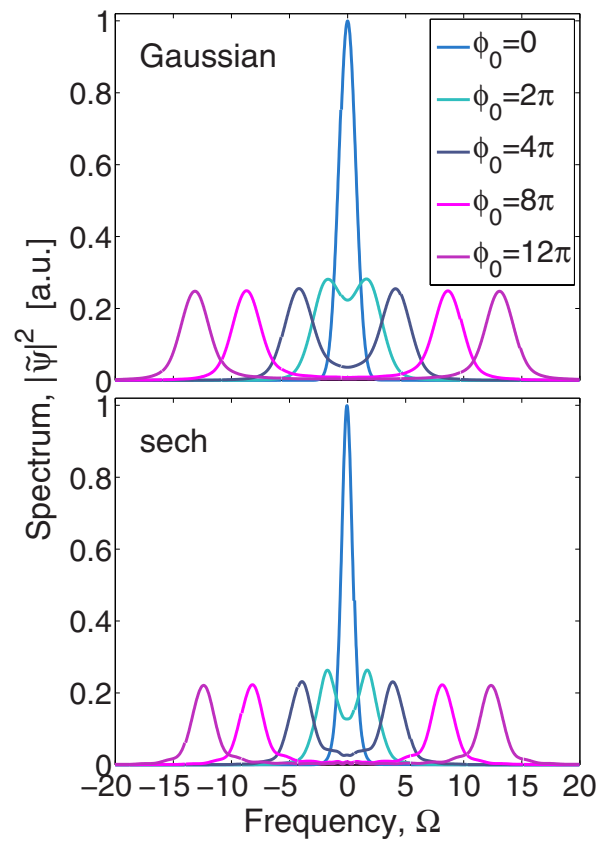

Fig. 1. (Color online) Initial pulse spectrum $\left(\phi_{0}=0\right)$ and XPMmodulated spectra for different values of $\phi_{0}$. Top, Gaussian pulse with $\eta=0.35$; bottom, sech pulse of the same FWHM temporal duration $(\eta=0.33)$. 


$$
\begin{aligned}
\left|\widetilde{\psi}_{\overline{+}}(\Omega)\right|^{2} \exp \left(-\epsilon^{2}\right) \\
\quad \simeq \frac{\pi u_{0}^{2} T^{2}}{2} \\
\quad \times \exp \left(-\epsilon^{2}\right)\left(1+\frac{2 \epsilon^{2}}{\pi}+\frac{2 \epsilon^{4}}{3 \pi}+\frac{7 \epsilon^{6}}{45 \pi}+\ldots\right) .
\end{aligned}
$$

The leading-order term in Eq. (2b) corresponds to a Gaussian shape. However, at this order, the energy contained in the side peak is half of the energy of the actual peak. At higher orders of the approximation, Eq. (2b) describes the central part of the actual peak with increased accuracy as well as captures an increased percentage of energy as compared with the actual peak.

The tolerable limits of the spectral splitting of the signal pulse to $\eta$ (varied from 0.01 to 1 ) and $\phi_{0}$ (from 0 to $16 \pi)$ are illustrated in Fig. 2. There, the region where splitting occurs is defined as the region where the extinction ratio-defined here as the ratio of the spectral intensity at $\Omega=0$ to the maximum intensity level-is less or equal to 0.1 .

The method of pulse doubling proposed in this paper is rather general and can be used to process signal pulses with various shapes. If, for instance, we consider an hyperbolic-secant-shaped (sech) initial pulse $\psi(0, t)$ $=u_{0} \operatorname{sech}(t / T)$, then an analytical formula analogous to Eq. (1) for the XPM-modulated pulse spectral amplitude can be derived as

$$
\begin{aligned}
\tilde{\psi}_{(}(\Omega)= & \tilde{\psi}_{0}(\Omega)+\tilde{\psi}_{-}(\Omega)+\tilde{\psi}_{+}(\Omega), \\
\tilde{\psi}_{0}(\Omega)= & 2 u_{0} T[\pi \operatorname{sech}(\pi \Omega / 2) \\
& -\frac{\exp ((1-i \Omega) / \eta)}{1-i \Omega} F(1,(1-i \Omega) / 2 ;(3 \\
& -i \Omega) / 2 ;-\exp (2 / \eta)) \\
& -\frac{\exp ((1+i \Omega) / \eta)}{1+i \Omega} F(1,(1+i \Omega) / 2 ;(3 \\
& +i \Omega) / 2 ;-\exp (2 / \eta))], \\
& +i(\Omega-\Delta \Omega)) / 2,0)+\frac{1}{2} \Psi((1+i(\Omega \\
\tilde{\psi}_{-}(\Omega)= & u_{0} T \exp \left(i \phi_{0}\right)[-B(-\exp (2 / \eta) ;(1 \\
& -\Delta \Omega)) / 4)-\frac{1}{2} \Psi((3+i(\Omega \\
& -\Delta \Omega) / 4)], \quad \tilde{\psi}_{+}(\Omega)=\tilde{\psi}_{-}(-\Omega) . \\
&
\end{aligned}
$$

In Eq. (3), $F(a, b ; c ; x), B(x ; a, b)$, and $\Psi(x)$ are the Gauss hypergeometric function, the incomplete beta function, and the digamma function, respectively. Eq. (3) looks

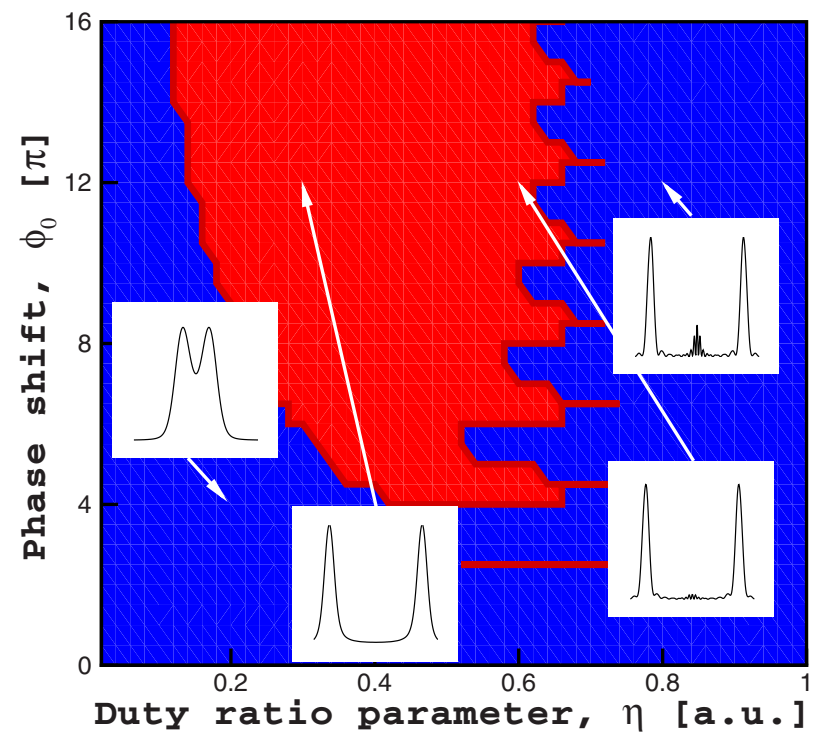

Fig. 2. (Color online) Limits of spectral copying in the plane duty ratio parameter-maximum XPM phase shift. Light area, copying occurs; dark area, copying does not occur. Insets, typical pulse spectra.

rather complicated, but the spectral features are similar to those of a Gaussian signal pulse, as can be seen in Fig. 1 (bottom). There is shown a similar plot to that in Fig. 1 (top) for a sech signal pulse with the same full width at half-maximum (FWHM) temporal duration as that of the Gaussian pulse (yielding $\eta=0.33$ ).

\section{TEMPORAL DOUBLING}

Next, we describe analytically the temporal pulse doubling that occurs upon propagation of the modulated (Gaussian) pulse in a linear dispersive medium. The temporal amplitude of the pulse after propagation over the distance $z$ is related to the temporal amplitude at the output of the XPM stage as

$$
\begin{aligned}
\psi(z, t)= & u_{0} \sqrt{i /(2 \pi D)} \int \mathrm{d} t^{\prime} \exp \left(-t^{\prime 2} /\left(2 T^{2}\right)\right) \exp \left(i \phi\left(t^{\prime}\right)-i(t\right. \\
& \left.\left.-t^{\prime}\right)^{2} /(2 D)\right)
\end{aligned}
$$

Here, $D=\beta_{2} z$ is the accumulated dispersion $\left(\beta_{2}\right.$ is standard GVD coefficient). The integral in this expression can easily be calculated to yield

$$
\begin{aligned}
\psi(\xi, \tau)= & \psi_{0}(\xi, \tau) \exp \left(-\frac{\tau^{2}(1+i \operatorname{sgn}(D) \xi)}{2\left(1+\xi^{2}\right)}\right) \\
& +\psi_{-}(\xi, \tau) \exp \left(-\frac{(\tau-\Delta \tau)^{2}}{2\left(1+\xi^{2}\right)}+i \Phi_{-}(\xi, \tau)\right) \\
& +\psi_{+}(\xi, \tau) \exp \left(-\frac{(\tau+\Delta \tau)^{2}}{2\left(1+\xi^{2}\right)}+i \Phi_{+}(\xi, \tau)\right), \\
\psi_{0}(\xi, \tau)= & \frac{u_{0}}{\sqrt{1-i \operatorname{sgn}(D) \xi}}\left[1+\frac{1}{2} \operatorname{erf}\left(X_{-}(\xi, \tau)\right)\right. \\
& \left.-\frac{1}{2} \operatorname{erf}\left(X_{+}(\xi, \tau)\right)\right]
\end{aligned}
$$




$$
\begin{aligned}
\psi_{-}(\xi, \tau)= & \frac{1}{2} \frac{u_{0}}{\sqrt{1-i \operatorname{sgn}(D) \xi}}\left[-\operatorname{erf}\left(X_{-}(\xi, \tau-\Delta \tau)\right)\right. \\
& \left.+\operatorname{erf}\left(X_{0}(\tau-\Delta \tau)\right)\right] \\
\psi_{+}(\xi, \tau)= & \psi_{-}(\xi,-\tau), \quad X_{0}(\xi, \tau) \\
= & \sqrt{\frac{i}{2}} \frac{\tau}{\sqrt{\operatorname{sgn}(D) \xi(1-i \operatorname{sgn}(D) \xi)}}, \\
X_{ \pm}(\xi, \tau)= & \sqrt{\frac{i}{2}}\left(\frac{\tau}{\sqrt{\operatorname{sgn}(D) \xi(1-i \operatorname{sgn}(D) \xi)}}\right. \\
& \left. \pm \eta^{-1} \frac{\sqrt{1-i \operatorname{sgn}(D) \xi}}{\sqrt{\operatorname{sgn}(D) \xi}}\right), \\
\Phi_{ \pm}(\xi, \tau)= & \phi_{0}-\frac{\tau^{2} \xi^{2} \mp 2 \tau \Delta \tau-\Delta \tau^{2}}{2 \operatorname{sgn}(D) \xi\left(1+\xi^{2}\right)}, \quad \xi=|D| / T^{2}, \quad \tau \\
= & t / T, \quad \frac{\Delta \tau=\operatorname{sgn}(D) \Delta \Omega \xi .}{2}
\end{aligned}
$$

In Eq. (4), $\xi$ and $\tau$ are a normalized distance and a normalized time, respectively, and $\operatorname{sgn}(x)$ is the sign function. It can be seen in Eq. (4) that in the case of normal disper$\operatorname{sion}(\operatorname{sgn}(D)=1)$, the pulse develops into a structure consisting of two identical pulses (optical copying) where the original pulse splits down the middle (for sufficiently small $\eta$ ). The two pulse parts separate in time while experiencing dispersive broadening. The relative velocity of separation $\Delta \tau$ is proportional to the separation $\Delta \Omega$ between the two peaks of the XPM-modulated pulse spectrum. It can also be inferred from Eq. (4) that pulse splitting and separation also occur in the case of anomalous dispersion $(\operatorname{sgn}(D)=-1)$ after an initial stage of pulse compression. The latter is controlled by the triangular pulse and dispersive medium parameters, and it is evidently expected as a result of compensation of the initial nonlinear (XPM-induced) pulse chirp in the anomalous dispersion regime of an optical fiber [2]. Figure 3 shows an example of pulse evolution in an ND fiber for $\phi_{0}$ $=12 \pi$ and $\eta=0.35$. The pulse intensity and chirp profiles at the beginning of propagation and at the distance $\xi=1$

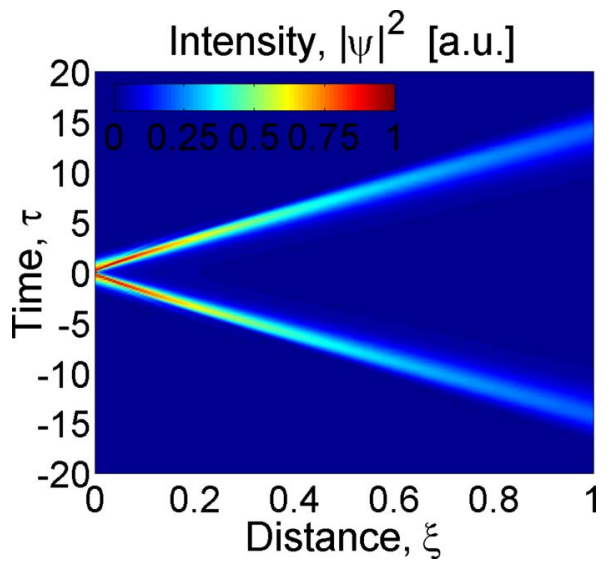

Fig. 3. (Color online) Evolution of the temporal intensity profile of the modulated pulse in a medium with normal dispersion for $\phi_{0}=12 \pi$ and $\eta=0.35$.
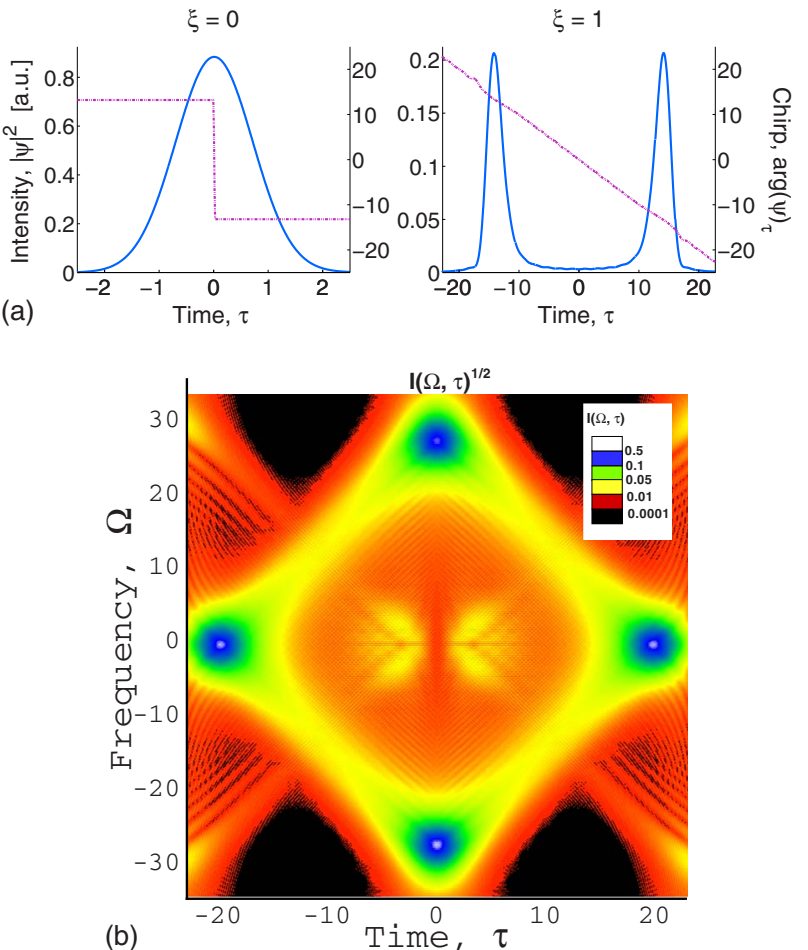

Fig. 4. (Color online) Top, intensity and chirp profiles of the pulse of Fig. 3 at $\xi=0$ and $\xi=1$. Bottom, pulse spectrogram at $\xi=1$.

and the pulse spectrogram at $\xi=1$ are shown in Fig. 4 . It is seen in Fig. 4 (top) that while at the fiber input the pulse chirp is a piecewise function, the two formed pulses acquire the same linear chirp upon propagation. Four maxima in the spectrogram of Fig. 4 (bottom) clearly indicate that the pulse is split in both time and frequency. Similar results can be derived also for other shapes of the initial signal pulse.

\section{CONCLUSION}

We have presented a novel technique of doubling optical pulses in both the frequency and time domains based on a combination of the nonlinear phase shift induced by a triangular pump pulse through XPM in a nonlinear Kerr medium and subsequent propagation in a dispersive medium. Exact analytical expressions for the XPMmodulated pulse spectrum and the pulse temporal intensity distribution have been derived. The presented technique could have important applications in devices performing optical signal manipulation and processing functions in telecomunications, lasers, and other areas of optics.

\section{REFERENCES}

1. S. Boscolo, A. I. Latkin, and S. K. Turitsyn, "Passive nonlinear pulse shaping in normally dispersive fiber systems," IEEE J. Quantum Electron. 44, 1196-1203 (2008).

2. G. P. Agrawal, Nonlinear Fiber Optics, 3rd ed. (Academic Press, 2001).

3. M. N. Islam, L. F. Mollenauer, R. H. Stolen, J. R. Simpson, and H.-T. Shang, "Cross-phase modulation in optical fibers," Opt. Lett. 12, 625-627 (1987). 
4. G. P. Agrawal, P. L. Baldeck, and R. R. Alfano, "Temporal and spectral effects of cross-phase modulation on copropagating ultrashort pulses in optical fibers," Phys. Rev. A 40, 5063-5072 (1989).

5. C. Yeh and L. Bergman, "Enhanced pulse compression in a nonlinear fiber by a wavelength division multiplexed optical pulse," Phys. Rev. E 57, 2398-2404 (1998).

6. L. Helczynski, B. Hall, D. Anderson, M. Lisak, A. Berntson, and M. Desaix, "Cross-phase modulation induced pulse splitting-the optical axe," Phys. Scr. T84, 81-84 (2000).

7. J. Li, B.-E. Olsson, M. Karlsson, and P. A. Andrekson, "OTDM demultiplexer based on XPM-induced wavelength shifting in highly nonlinear fiber," IEEE Photonics Technol. Lett. 15, 1770-1772 (2003).

8. Y. Park, M. H. Asghari, T.-J. Ahn, and J. Azaña, "Transform-limited picosecond pulse shaping based on temporal coherence synthesization," Opt. Express 15 , 9584-9599 (2007).

9. P. Petropoulos, M. Ibsen, A. D. Ellis, and D. J. Richardson, "Rectangular pulse generation based on pulse reshaping using a superstructured fiber Bragg grating," J. Lightwave Technol. 19, 746-752 (2001).

10. F. Parmigiani, P. Petropoulos, M. Ibsen, and D. J. Richardson, "Pulse retiming based on XPM using parabolic pulses formed in a fiber Bragg grating," IEEE Photonics Technol. Lett. 18, 829-831 (2006).

11. F. Parmigiani, C. Finot, K. Mukasa, M. Ibsen, M. A. F Roelens, P. Petropoulos, and D. J. Richardson, "Ultra-flat SPM-broadened spectra in highly nonlinear fiber using parabolic pulses formed in a fiber Bragg grating," Opt. Express 14, 7617-7622 (2006).

12. F. Parmigiani, M. Ibsen, T. T. Ng, L. Provost, P. Petropoulos, and D. J. Richardson, "An efficient wavelength converter exploiting a grating based saw-tooth pulse shaper," IEEE Photonics Technol. Lett. 20, 1461-1463 (2008).

13. B. H. Kolner, "Space-time duality and the theory of temporal imaging," IEEE J. Quantum Electron. 30 1951-1963 (1994).

14. M. Nakazawa and T. Hirooka, "Distortion-free optical transmission using time-domain optical Fourier transformation and transform-limited optical pulses," J. Opt. Soc. Am. B 22, 1842-1855 (2005).

15. R. S. Bhamber, S. Boscolo, A. I. Latkin, and S. K. Turitsyn, "All-optical TDM to WDM signal conversion and partial regeneration using XPM with triangular pulses," in Proceedings of the 34th European Conference on Optical Communication (ECOC 2008), paper Th.1.B.2 (2008). 